\title{
Revisão de escopo sobre o instrumento “Occupational Performance History Interview - II”: perspectivas para o seu uso no Brasil
}

\section{Scoping review about the "Occupational Performance History Interview - II" instrument: perspectives for use in Brazil}

Julia Andreza Gorla ${ }^{a}$ (D), Cláudia Maria Simóes Martinez ${ }^{a}$ (D), Mirela de Oliveira Figueiredo ${ }^{a}$ (D), Daniel Marinho Cezar da Cruz ${ }^{\mathrm{a}}$ (D)

${ }^{a}$ Universidade Federal de São Carlos - UFSCar, São Carlos, SP, Brasil.

Como citar: Gorla, J. A., Martinez, C. M. S., Figueiredo, M. O., \& Cruz, D. M. C. (2021). Revisão de escopo sobre o instrumento "Occupational Performance History Interview - II": perspectivas para o seu uso no Brasil. Cadernos Brasileiros de Terapia Ocupacional, 29, e2807. https://doi.org/10.1590/2526-

8910.ctoAR2123

\begin{abstract}
$\underline{\text { Resumo }}$
Introduçáo: O instrumento "Occupational Performance History Interview - II" (OPHI-II) foi elaborado para se configurar como avaliação para terapeutas ocupacionais que atuam sob a perspectiva do Modelo de Ocupaçáo Humana (MOHO). Por meio de entrevista semi-estruturada, coleta informaçóes sobre o desempenho ocupacional do cliente na busca de obter efetividade do planejamento e processo terapêutico. Objetivo: Investigar a produção de conhecimento da terapia ocupacional relativa à utilização do OPHI-II quanto à finalidade de uso e modos de aplicação, discutindo as contribuiçôes para a prática do terapeuta ocupacional e a perspectiva de uso no Brasil. Método: Trata-se de uma revisão de escopo da literatura, realizada nas bases de dados Web of Science, Scopus e Biblioteca Virtual em Saúde, somada aos artigos sugeridos no site e livro do MOHO. Resultados: Localizaram-se 14 periódicos, totalizando 26 publicações com o uso do OPHI-II, a maioria delas posteriores a 2010, sendo a mais recente de 2017. Identificaram-se sete países de estudo, com destaque para Estados Unidos e Austrália, sem pesquisas conduzidas no Brasil. Em sua maioria, a abordagem escolhida foi a qualitativa, com metodologia baseada na análise temática do discurso. Há diversidade em relação à populaçáo estudada e ao modo de aplicação do OPHI-II, podendo combinar ou não os seus três elementos (entrevista, curva narrativa e escalas). Conclusáo: $\mathrm{O}$ OPHI-II se mostrou como um instrumento relevante tanto na prática clínica como para a pesquisa, o qual possibilita a combinação de outras escalas de avaliação e tem sido utilizado em diversas populaçóes e áreas do campo da terapia ocupacional.
\end{abstract}

Palavras-chave: Atividades Cotidianas, Avaliação de Resultados de Intervençôes Terapêuticas, Prática Clínica Baseada em Evidências, Terapia Ocupacional. 


\begin{abstract}
$\underline{\text { Abstract }}$
Introduction: The "Occupational Performance History Interview” - II (OPHI-II) was developed to be the initial assessment for occupational therapists who work under the perspective of the Model of Human Occupation (MOHO). This instrument can be applied as an interview script to gather information about a patient's occupational performance, enabling the therapeutic plan and measuring its effectiveness. Objective: To review the publications about the OPHI-II in occupational therapy regarding the purpose and methods of its use, and to discuss its contributions to clinical practice and its perspectives for application in Brazil. Method: This is a scoping review performed in the databases: Web of Science, Scopus, and Virtual Health Library. We also search in grey literature such as the MOHO book and its website. Results: We identified that in 14 of overall 26 publications using the OPHI-II, most of them were published after 2010, and the newest was in 2017. Seven countries were identified in those publications, mainly the United States and Australia, but no Brazilian study was observed. Mostly, the chosen approach was qualitative, based on thematic content analysis. The population studied was diverse and also the methods of application of the OPHIII, combining or not its three elements (i.e. interview, narrative curve, and scales). Conclusion: OPHI-II shows to be a relevant instrument both in clinical practice and research, which allows the combination of other assessment scales and has been used with a range of clients in fields of practice in occupational therapy.
\end{abstract}

Keywords: Activities of Daily Living, Evaluation of Results of Therapeutic Interventions, Evidence-Based Practice, Occupational Therapy.

\title{
Introduçáo
}

O uso de um modelo para fundamentar o raciocínio terapêutico ocupacional fornece ao profissional um embasamento para pensar na avaliação, no planejamento e na implementaçáo de um plano terapêutico. Conhecer os conceitos teóricos utilizados por um modelo facilita a sua compreensão, bem como sua aplicação (Keller \& Forsyth, 2004).

Segundo o Modelo de Ocupação Humana (MOHO), o termo "occupational performance", em livre tradução para o português como "desempenho ocupacional", refere-se às escolhas e aos desempenhos em ocupaçóes significativas, seja no trabalho, lazer ou nas atividades de autocuidado. O desempenho ocupacional é formado por unidades de ação que, ao serem executadas, levam à conclusão de uma atividade desejada de modo completo e coerente (Pablo et al., 2017).

Ao coletar informaçóes sobre o histórico do desempenho ocupacional, o terapeuta ocupacional poderá ter acesso a informações como os hábitos e as motivaçóes envolvidos no engajamento ocupacional de seu cliente, bem como às suas dimensóes da participação no fazer (Pablo, 2015; Pablo et al., 2017). O conhecimento sobre esses aspectos fornece um maior embasamento no planejamento terapêutico do cliente e uma maior aproximação e vínculo, porque facilita o entendimento da pessoa enquanto um ser ocupacional. Ainda, essa coleta sobre a história convida a pessoa a sentir e pensar sobre 
o seu fazer, incluindo-a de forma participativa em todo o seu processo de tratamento; uma premissa da prática centrada no cliente.

Pensando na importância da dimensão do desempenho ocupacional, a Associação Americana de Terapia Ocupacional (AOTA) encomendou uma avaliação que pudesse se tornar padrão da prática clínica profissional nos Estados Unidos. Foi então que, em 1989, criou-se a primeira versão do "Occupational Performance History Interview" (OPHI), a qual foi validada para o Brasil em 1998 (Benetton \& Lancman, 1998). Embora o OPHI tenha sido adaptado e validado para o Brasil, são desconhecidas outras publicaçôes quanto ao uso desse instrumento no país. Em 2004, uma segunda versão foi publicada, a qual se encontra em processo de adaptação para a cultura brasileira (Kielhofner et al., 2004).

O OPHI, desde sua primeira versão, consiste em uma entrevista semiestruturada voltada para a história de vida do cliente, reunindo informaçóes sobre o seu desempenho ocupacional passado e atual, nas áreas de trabalho, lazer e autocuidado. É uma entrevista que pode ser utilizada para avaliar clientes diversos, sendo considerada genérica (Benetton \& Lancman, 1998). Foi elaborada para ser uma avaliaçáo inicial, com o intuito de melhorar a compreensão do terapeuta sobre o cliente e guiar o planejamento da intervenção terapêutica ocupacional (Kielhofner et al., 2001).

Pelo fato de seu foco estar centrado na história de vida do cliente, o instrumento, em sua segunda versão, mostra-se um importante aliado para a intervenção da terapia ocupacional, bem como se baseia na teoria do $\mathrm{MOHO}$, uma vez que consegue abarcar os conceitos de adaptação ocupacional (incluindo identidade e competência), volição (causação pessoal, valores e interesses), habituação (hábitos e papéis) e ainda a participação nos papéis (Turpin \& Iwama, 2011).

O OPHI-II é um instrumento de avaliação que contém três partes, a saber: 1) entrevista semiestruturada, que explora a história de vida do cliente; 2) escalas avaliativas, as quais mensuram a identidade e a competência ocupacional do cliente, bem como o impacto causado pelo seu ambiente; e 3) a curva narrativa que esboça a história de vida do cliente ao longo do tempo e em relação a um evento crítico (Hemmingsson et al., 2017).

A validade interna da OPHI-II já foi comprovada, bem como a sua possibilidade de aplicação em uma ampla gama de pessoas de diferentes culturas (Kielhofner et al., 2001). Uma vez que o OPHI-II já passou por um processo de revisão e comprovação de sua validade em países como Estados Unidos, Finlândia, Austrália, Islândia, Bélgica, entre outros (Kielhofner et al., 2001), considera-se que uma análise da produção de conhecimento quanto à sua utilização poderá fornecer subsídios para compreensão da aplicação do instrumento na prática da terapia ocupacional.

A partir desta consideração, o presente estudo teve por objetivo analisar a produção de conhecimento sobre o OPHI-II quanto à sua finalidade de uso e aos modos de aplicação, discutindo as contribuiçóes do instrumento para a prática do terapeuta ocupacional e a perspectiva de uso no Brasil.

Para tanto, este trabalho foi conduzido a partir das seguintes perguntas investigativas: Com quais populaçóes foi utilizado o instrumento OPHI-II?; Quais as finalidades para o uso do OPHI-II?; e Quais os tipos de pesquisa em que o OPHI-II foi utilizado?

Para além das perguntas, procurou-se verificar sobre a utilização do OPHI-II em conjunto com outros instrumentos padronizados, conhecer os desenhos metodológicos 
utilizados para a coleta e análise dos dados obtidos pela aplicação do OPHI-II, além de identificar indicadores bibliométricos, como o ano da produção, o país no qual a pesquisa foi desenvolvida, a base de dados na qual o estudo pôde ser encontrado e as revistas nas quais os estudos foram publicados.

\section{Método}

Trata-se de uma revisão de escopo, tipo de revisão que possibilita o mapeamento das fontes, evidências e conceitos que subsidiam uma área de conhecimento. Em conjunto, permite a identificação das lacunas existentes nesta área pesquisada (Arksey \& O’Malley, 2005). A revisão de escopo é considerada um procedimento transversal que, preferencialmente, aplica-se a áreas que não foram revisadas anteriormente (Arksey \& O’Malley, 2005), como é o caso do presente estudo.

Para a realização desta revisão, foram adotados os parâmetros estabelecidos por Arksey \& O’Malley (2005), e complementados por Donato \& Donato (2019), O’Brien et al. (2016), Peters et al. (2015), Colquhoun et al. (2014) e Tricco et al. (2016), sendo realizadas as cinco etapas:

1) elaboração das perguntas de pesquisa;

2) identificação dos estudos por meio de diferentes fontes;

3) composição da amostra com base nos critérios de busca e inclusão/exclusão;

4) extração de informações relativas às perguntas de pesquisa;

5) análise numérica e temática dos dados, descrição e discussão.

\section{Estratégias de busca}

A busca dos estudos foi realizada a partir dos artigos que constam no site do MOHO (Model of Human Occupation Clearinghouse, 2020) e nas fontes de indexação: Biblioteca Virtual de Saúde, Web of Science e Scopus, bem como pelas indicaçóes contidas na última edição do livro Kielhofner's Model of Human Occupation: theory and application (Taylor, 2017), já que, a despeito da vasta publicação sobre o modelo, ainda é nos livros que se concentra uma maior quantidade de informaçóes atualizadas sobre seus conceitos, evolução da teoria, assim como as evidências existentes.

Para a busca nas fontes, a fim de alcançar o maior número de estudos publicados sobre o OPHI-II, foram utilizadas seis combinaçóes booleanas, constantes no título, resumo ou palavras-chave dos estudos, feitas da mesma forma e na mesma ordem em todas as fontes:

10 "Occupational Performance History Interview - II"

2o "OPHI-II"

3o "Occupational Performance History Interview - II" OR "OPHI-II"

40 "Occupational Performance History Interview - IT" AND "Model of Human Occupation"

5o "OPHI-II" AND “Model of Human Occupation" 


\section{$6^{\circ}$ "Occupational Performance History Interview - Ir" OR “OPHI-II" AND “Model of Human Occupation"}

Mesmo admitindo-se a importância e popularidade do uso de expressões indexadas em bases de dados, definiu-se que as palavras-chave para a presente revisão não deveriam se distanciar do fenômeno a ser investigado (um instrumento de avaliação - OPHI-II parte de um modelo - MOHO), o qual tem uma terminologia bastante específica. Assim, optou-se pelo uso de expressóes que fossem ao encontro do objetivo do estudo, mesmo que estas não estivessem listadas como descritores em bancos de dados.

\section{Critérios de inclusáo}

- Documentos que utilizaram o OPHI-II e que forneciam ao menos uma informação sobre esta utilização, como: população, finalidade, resultados obtidos e função para a prática terapêutica ocupacional.

- Documentos disponíveis na íntegra, escritos em inglês, espanhol e português, em forma de artigo científico, capítulo de livro ou livro inteiro.

- Documentos resultantes de pesquisas quantitativas ou qualitativas e com qualquer desenho de estudo (estudo controlado randomizado, grupo único pré-pós, coorte, caso-controle, pesquisas, revisóes, teoria narrativa, estudos de caso ou relatos de experiência).

\section{Critérios de exclusão}

Foram excluídos editoriais e anais de congressos, documentos que utilizaram a primeira versão do OPHI e aqueles que não utilizaram o OPHI-II em seu formato original, por exemplo, que utilizaram esse instrumento apenas como base para elaborar uma nova avaliação.

A busca nas bases de dados e site foi realizada em fevereiro de 2020, sem janelamento de tempo, para que todas as publicações pudessem ser consideradas. Após a retirada dos artigos duplicados, foi feita a leitura dos estudos na íntegra, a fim de cumprir com os critérios de elegibilidade da revisão. A Figura 1 ilustra o processo de busca e composição da amostra final de textos, realizado pela autora principal desta revisão.

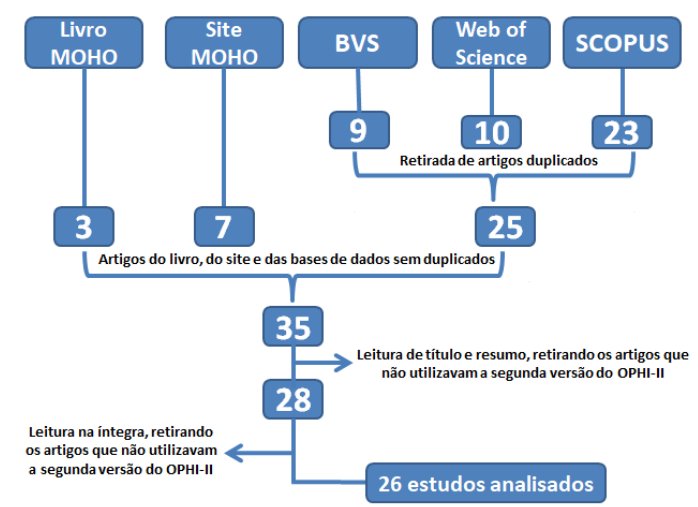

Figura 1. Processo de busca e composição da amostra final de textos. 


\section{Resultados}

Inicialmente, serão apresentados os resultados referentes aos artigos selecionados pelos periódicos e fontes de indexação. $\mathrm{Na}$ sequência, apresentam-se a distribuição das publicaçôes ao longo dos anos e a identificação dos seus respectivos países. Dados sobre as abordagens metodológicas e a população estudada são descritas na sequência. Por fim, são apresentados os resultados referentes aos objetivos dos estudos, uso e finalidade do OPHI-II com a utilização de outros instrumentos de avaliação.

Após a aplicação dos critérios de inclusão e exclusão, foram selecionados 26 estudos para análise, publicados em 14 periódicos, dos quais se destacam o Australian Occupational Therapy Journal, o Scandinavian Journal of Occupational Therapy, seguido do British Journal of Occupational Therapy e do Occupational Therapy in Health Care, por terem publicado 15 dos 26 artigos incluídos na revisão. Em relação às fontes de indexação, nota-se que a Scopus foi a base que mais conteve artigos indexados, seguida pela base Web of Science (Tabela 1).

Tabela 1. Distribuição dos artigos científicos por periódicos e fontes de indexaçâoo ( $n=26)$.

\begin{tabular}{|c|c|c|}
\hline Nome do artigo/Autores/Ano & Periódico & Fontes de indexaçáo \\
\hline \multirow{3}{*}{$\begin{array}{l}\text { Psychometric Properties of the Second Version } \\
\text { of the Occupational Performance History } \\
\text { Interview (OPHI-II) } \\
\text { (Kielhofner et al., 2001) }\end{array}$} & \multirow{3}{*}{$\begin{array}{c}\text { The American Journal of } \\
\text { Occupational Therapy }\end{array}$} & $B V S$ \\
\hline & & Web of Science \\
\hline & & SCOPUS \\
\hline $\begin{array}{l}\text { Illness experiences and occupations of people } \\
\text { with chronic fatigue syndrome } \\
\text { (Gray \& Fossey, 2003) }\end{array}$ & $\begin{array}{c}\text { Australian Occupational } \\
\text { Therapy Journal }\end{array}$ & SCOPUS \\
\hline $\begin{array}{l}\text { Being in a secure forensic psychiatric unit: } \\
\text { every day is the same, killing time or make } \\
\text { the most of it } \\
\text { (Farnworth et al., 2004) }\end{array}$ & $\begin{array}{c}\text { British Journal of } \\
\text { Occupational Therapy }\end{array}$ & SCOPUS \\
\hline $\begin{array}{l}\text { Life skill service needs: perspectives of } \\
\text { bomeless youth } \\
\text { (Aviles \& Helfrich, 2004) }\end{array}$ & $\begin{array}{c}\text { Journal of Youth and } \\
\text { Adolescence }\end{array}$ & Site do $\mathrm{MOHO}$ \\
\hline $\begin{array}{l}\text { Caring and daily life: occupational } \\
\text { experiences of women living with sons } \\
\text { diagnosed with schizophrenia } \\
\text { (Chaffey \& Fossey, 2004) }\end{array}$ & $\begin{array}{c}\text { Australian Occupational } \\
\text { Therapy Journal }\end{array}$ & SCOPUS \\
\hline $\begin{array}{l}\text { Occupational narratives and the therapeutic } \\
\text { process } \\
\text { (Goldstein et al., 2004) }\end{array}$ & $\begin{array}{c}\text { Australian Occupational } \\
\text { Therapy Journal }\end{array}$ & Livro \\
\hline Therapist's and client's perceptions of the & Occupational Therapy in & $B V S$ \\
\hline $\begin{array}{l}\text { occupational performance history interview } \\
\text { (Apte et al., 2005) }\end{array}$ & Health Care & SCOPUS \\
\hline The construction of key-forms for obtaining & Occupational Therapy & Web of Science \\
\hline $\begin{array}{l}\text { instantaneous measures from the } \\
\text { occupational performance history rating } \\
\text { scales (Kielhofner et al., 2005) }\end{array}$ & $\begin{array}{c}\text { Journal of Research: } \\
\text { Occupational, Participation } \\
\text { and Health }\end{array}$ & SCOPUS \\
\hline & WORK & $B V S$ \\
\hline
\end{tabular}


Tabela 1. Continued..

\begin{tabular}{lcc}
\hline \multicolumn{1}{c}{ Nome do artigo/Autores/Ano } & Periódico & Fontes de indexaçáo \\
\hline Occupational Identity, occupational & & \\
competence and occupational settings & & SCOPUS \\
(environment): influences on return to work & & \\
in men living with HIVIAIDS & & \\
(Braveman et al., 2006)
\end{tabular}

The occupational performance history interview in community mental health care management: consumer and occupational therapist perspectives

\author{
Web of Science
}

Australian Occupational Therapy Journal

SCOPUS

(Ennals \& Fossey, 2007)

Narrative slope as a predictor of work and other occupational participation

(Levin et al., 2007)

Scandinavian Journal of

Occupational Therapy

SCOPUS

Addressing the needs of elderly clients whose lives have been compounded by traumatic bistories

Occupational Therapy in Health Care

SCOPUS

(Ziv \& Roitman, 2008)

Using the OPHI-II to support people with mental illness in their recovery

(Ennals \& Fossey, 2009)

Occupational Therapy in

Mental Health

SCOPUS

The road to recovery: experience and occupational lives of Icelandic women with breast cancer

Occupational Therapy in Health Care

SCOPUS

(Palmadottir, 2009)

Exploring occupational adaptation through the lives of women with multiple sclerosis (Cabill et al., 2010)

British Journal of

Occupational Therapy

Web of Science

SCOPUS

Time use in forensic psychiatry: a naturalist inquiry into two forensic patients in Australia

International Journal of

Forensic Mental Health

SCOPUS

(O'Connel et al., 2010)

The role of occupational participation and environment among Icelandic women with breast cancer: a qualitative study (Palmadottir, 2010)

Occupational Identity: exploring the narratives of three men living with AIDS (Braveman \& Helfrich, 2001)

Exploring the impact of sleep apnoea on daily life and occupational engagement (O'Donoghue \& McKay, 2012)

Scandinavian Journal of

Occupational Therapy

Web of Science

SCOPUS

Participating in diagnostic experience: adults puitelanpucopisifhicatric disorders (Sandall et al., 2013)

Improved occupational performance of young adults with a physical disability after a vocational rehabilitation intervention (Verboef et al., 2014)

Journal of Occupational Science

SCOPUS

British Journal of

Occupational Therapy

Web of Science

Scandinavian Journal of

Occupational Therapy

BVS

Web of Science

SCOPUS

BVS

Journal of Occupational

Rehabilitation

Web of Science

SCOPUS 


\begin{tabular}{lcc}
\hline \multicolumn{1}{c}{ Nome do artigo/Autores/Ano } & Periódico & Fontes de indexaçáo \\
\hline $\begin{array}{l}\text { Mothering role identity and competence } \\
\text { among parenting and pregnant homeless } \\
\text { adolescents }\end{array}$ & $\begin{array}{c}\text { Journal of Occupational } \\
\text { (Levin \& Helfrich, 2004) }\end{array}$ & Science \\
\hline $\begin{array}{l}\text { "Our child's significant disability shapes our } \\
\text { lives": experiences of family social }\end{array}$ & & \\
$\begin{array}{l}\text { participation } \\
\text { (Davey et al., 2015) }\end{array}$ & Disability and Rehabilitation & Web of Science \\
\hline $\begin{array}{l}\text { Secondary Health conditions experienced by } \\
\text { people with spinal cord injury within } \\
\text { community living: implications for a } \\
\text { national disability insurance scheme } \\
\text { (Callaway et al., 2015) }\end{array}$ & Australian Occupational & SCOPUS \\
\hline $\begin{array}{l}\text { Male-to-female transitions: implications for } \\
\text { occupational performance, health and life } \\
\text { satisfaction }\end{array}$ & Therapy Journal & Site MOHO \\
(Bar et al., 2016) & $\begin{array}{c}\text { Canadian Journal of } \\
\text { Occupational identity of adolescents with }\end{array}$ & Site MOHO \\
$\begin{array}{l}\text { ADHD: a mixed methods study } \\
\text { (Levanon-Erez et al., 2017) }\end{array}$ & Scandinavian Journal of & \\
\hline
\end{tabular}

Com relação aos anos de publicação, 15 artigos datam anteriormente ao ano de 2010, sendo que, em 2002, não houve nenhuma publicação e, em 2004, concentra-se o maior número de estudos com o OPHI-II. Após 2010, foram encontrados 11 artigos, sendo que o ano de 2014 não teve nenhum e o mais recente data de 2017. A Figura 2 traz a distribuição da quantidade de artigos publicados ao longo dos anos.

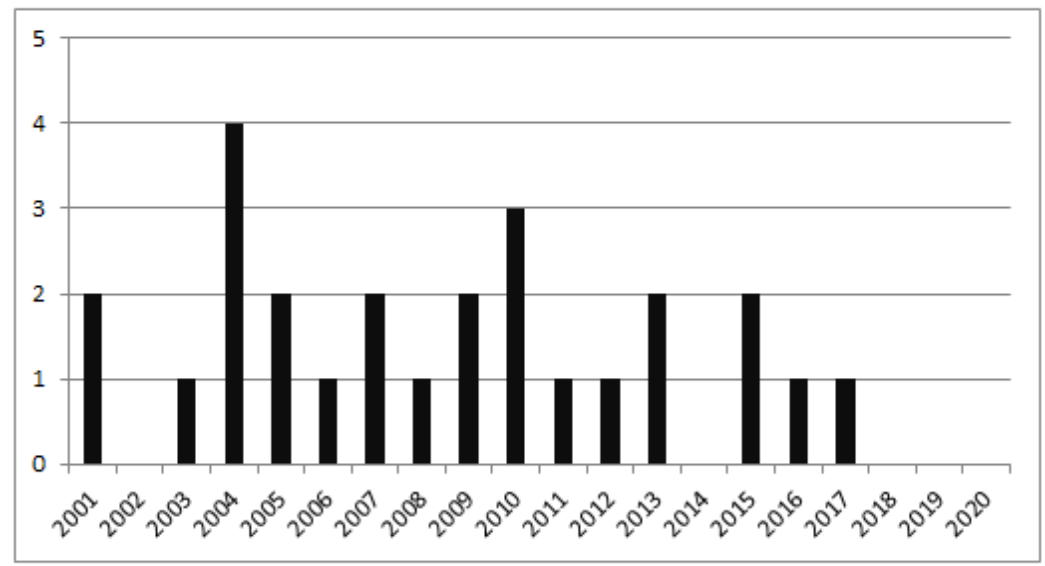

Figura 2. Distribuição da quantidade de artigos publicados ao longo dos anos.

As pesquisas foram conduzidas em 7 países, com destaque para 9 estudos realizados nos Estados Unidos e 8 na Austrália. Houve ainda publicações realizadas em Israel $(n=3)$, na Irlanda $(n=2)$, Islândia $(n=2)$, Holanda $(n=1)$ e Suécia $(n=1)$. Também se pôde notar 
que, de todos os autores envolvidos, alguns compuseram a equipe de diferentes publicaçôes: Kielhofner $(n=6)$, Fossey $(n=6)$, Braveman $(n=4)$, Farnworth $(n=3)$ e Helfrich $(\mathrm{n}=4)$.

As pesquisas foram, em sua maioria (17), de abordagem qualitativa, com metodologia de tratamento dos dados baseada na análise temática do discurso. Houve ainda 5 estudos quantitativos e 4 com metodologia mista. Naqueles com abordagem quantitativa, utilizou-se de análise descritiva simples, a partir das pontuaçóes das escalas do OPHI-II ou de outros instrumentos utilizados em combinação.

Com relação à população estudada, as amostras foram variadas, abarcando adolescentes em situação de vulnerabilidade social (2) ou com diagnóstico de Transtorno de Deficit de Atenção e Hiperatividade TDAH (1), mulheres com câncer de mama (2), adultos em cuidados quanto à saúde mental (8), adultos com disfunção física em reabilitação física (4), adultos com disfunção física em processo de reabilitação profissional (2), idosos (1), pessoas com diagnóstico de Síndrome da Imunodeficiência Adquirida (HIV/AIDS) (4), de apneia obstrutiva do sono (1) e síndrome da fadiga crônica (1), população transgênero (1), cuidadores (2), indivíduos sem qualquer diagnóstico ou condição de saúde (1) e terapeutas ocupacionais (4). Cabe notar que alguns estudos $(n=5)$ tiveram amostra com mais de um dos grupos citados, como o estudo de Kielhofner et al. (2001), por exemplo, o qual utilizou, além de terapeutas ocupacionais, participantes com diferentes condições de saúde (sem nenhum diagnóstico médico, com incapacidade física e com transtorno mental), e o estudo de Ennals \& Fossey (2007), com a participação de terapeutas ocupacionais e seus clientes atendidos em um serviço de saúde mental.

Sobre o uso do OPHI-II, 14 estudos utilizaram apenas a entrevista, a fim de coletar a história de vida; 5 preencheram também a curva narrativa; 4 estudos pontuaram as escalas e 3 combinaram os três elementos do OPHI-II (entrevista, curva narrativa e escalas). Tais dados podem ser observados na Tabela 2, a qual também apresenta a finalidade da utilização do OPHI-II, que se mostrou relevante não só para analisar os constructos de suas 3 escalas (identidade, competência e ambiente), mas também outros importantes elementos constituintes do MOHO. De todos os 26 estudos, 16 combinaram outros instrumentos de coleta de dados, com destaque para o diário de campo, feito em 10 estudos como forma de complementar as informaçốes coletadas com as entrevistas do OPHI-II.

Nota-se que o OPHI-II foi utilizado por estudos com populaçóes variadas, tanto em diagnóstico quanto em idade.

Os objetivos se concentram na compreensão de conceitos do $\mathrm{MOHO}$, como adaptação ocupacional, identidade e desempenho ocupacional, o uso do tempo e a experiência do cuidado. Observa-se que os autores optaram por utilizar o OPHI-II em conjunto com outros meios de avaliação, seguindo diferentes metodologias de coleta e análise de dados. 
Tabela 2. Uso e finalidade do OPHI-II com a utilização de outros instrumentos de avaliaçấo.

\begin{tabular}{|c|c|c|}
\hline Uso do OPHI-II & Finalidade do OPHI-II & Outros instrumentos \\
\hline Entrevista + escalas & $\begin{array}{l}\text { Investigar sobre a identidade ocupacional de } \\
\text { adolescentes com TDAH }\end{array}$ & $\begin{array}{l}\text { Conner's Ratings Scales- } \\
\text { Revised (CRS-R) }\end{array}$ \\
\hline Entrevista + escalas & $\begin{array}{l}\text { Investigar identidade, competência e ambiente } \\
\text { ocupacional, junto ao desempenho ocupacional } \\
\text { de mulheres transgênero. }\end{array}$ & $\begin{array}{c}\text { Questionário demográfico, } \\
\text { Short Form Health Survey } \\
36 \text { (SF-36), } \\
\text { Satisfaction With Life Scale } \\
\text { (SWLS) }\end{array}$ \\
\hline Entrevista & $\begin{array}{l}\text { Compreender o impacto das condiçóes } \\
\text { secundárias de saúde para a vida em comunidade } \\
\text { de pessoas que sofreram lesão medular }\end{array}$ & $\begin{array}{l}\text { Questionário demográfico, } \\
\text { The Spinal Cord Injury } \\
\text { Secondary Conditions Scale } \\
\text { (SCISCS), } \\
\text { Diário de campo }\end{array}$ \\
\hline Entrevista & $\begin{array}{l}\text { Compreender a participação social das famílias } \\
\text { de jovens com deficiência física }\end{array}$ & Diário de campo \\
\hline Entrevista & $\begin{array}{l}\text { Compreender o senso de competência e } \\
\text { identidade de mães adolescentes em situação de } \\
\text { rua }\end{array}$ & Não \\
\hline $\begin{array}{l}\text { Entrevista }+ \text { curv } \\
\text { narrativa +escalas }\end{array}$ & $\begin{array}{l}\text { Conhecer as necessidades e realizar intervençóes } \\
\text { terapêuticas mais assertivas com adultos com } \\
\text { deficiência física em processo de reabilitação } \\
\text { profissional }\end{array}$ & $\begin{array}{l}\text { Canadian Occupational } \\
\text { Performance Measure } \\
\text { (COPM) }\end{array}$ \\
\hline Entrevista & $\begin{array}{l}\text { Descrever experiências de vida de adultos com } \\
\text { transtornos neuropsiquiátricos }\end{array}$ & Não \\
\hline Entrevista & $\begin{array}{l}\text { Descrever experiências ocupacionais de } \\
\text { indivíduos com apneia obstrutiva do sono }\end{array}$ & Diário de campo \\
\hline $\begin{array}{l}\text { Entrevista + curva } \\
\text { narrativa }\end{array}$ & $\begin{array}{l}\text { Compreender a identidade ocupacional de } \\
\text { adultos com HIV/AIDS }\end{array}$ & Não \\
\hline Entrevista & $\begin{array}{l}\text { Ilustrar a participaçáo ocupacional e a influência } \\
\text { do ambiente na percepçáo de saúde e bem-estar } \\
\text { de mulheres com câncer de mama }\end{array}$ & Não \\
\hline Entrevista & $\begin{array}{l}\text { Compreender o uso do tempo de sujeitos } \\
\text { vivendo em manicômio judicial }\end{array}$ & $\begin{array}{l}\text { Australian Time Use } \\
\text { Survey, } \\
\text { Diário de campo }\end{array}$ \\
\hline Entrevista & $\begin{array}{l}\text { Explorar a adaptaçáo ocupacional de mulheres } \\
\text { com esclerose múltipla }\end{array}$ & $\begin{array}{c}\text { Occupational } \\
\text { Questionnaire, } \\
\text { Role Checklist, } \\
\text { Modified Interest Checklist }\end{array}$ \\
\hline Entrevista & $\begin{array}{l}\text { Explorar as experiências ocupacionais a fim de } \\
\text { promover adaptaçáo ocupacional de mulheres } \\
\text { com câncer de mama }\end{array}$ & Diário de campo \\
\hline $\begin{array}{l}\text { Entrevista + curva } \\
\text { narrativa }\end{array}$ & $\begin{array}{l}\text { Entender experiências de vida e apoiar o } \\
\text { processo terapêutico de pacientes com doenças } \\
\text { mentais. }\end{array}$ & Não \\
\hline
\end{tabular}


Tabela 2. Continued...

\begin{tabular}{|c|c|c|}
\hline Uso do OPHI-II & Finalidade do OPHI-II & Outros instrumentos \\
\hline $\begin{array}{l}\text { Entrevista + curva } \\
\text { narrativa + escalas }\end{array}$ & $\begin{array}{l}\text { Embasar e guiar o processo terapêutico de idosas } \\
\text { com histórias de vida traumáticas a partir do } \\
\text { MOHO. }\end{array}$ & Não \\
\hline $\begin{array}{l}\text { Entrevista + curva } \\
\text { narrativa }\end{array}$ & $\begin{array}{l}\text { Avaliar a capacidade da curva narrativa em } \\
\text { prever a participaçáo ocupacional e o } \\
\text { engajamento no trabalho de pessoas com } \\
\text { diagnóstico de HIV/AIDS }\end{array}$ & $\begin{array}{l}\text { Avaliação demográfica, } \\
\text { Sign and Symptom } \\
\text { Checklist-HIV (SSC-HIV) }\end{array}$ \\
\hline Entrevista & $\begin{array}{l}\text { Explorar a utilidade do uso do OPHI-II sob a } \\
\text { perspectiva de TOs e seus clientes atendidos na } \\
\text { área de saúde mental }\end{array}$ & Diário de campo \\
\hline $\begin{array}{l}\text { Entrevista + curva } \\
\text { narrativa + escalas }\end{array}$ & $\begin{array}{l}\text { Descrever as mudanças ocorridas no senso de } \\
\text { competência e identidade ocupacional e } \\
\text { identificar as dificuldades nos ambientes } \\
\text { ocupacionais de homens com HIV/AIDS que } \\
\text { retornaram ao trabalho }\end{array}$ & Não \\
\hline Entrevista + escalas & Desenvolver as "key-forms" do OPHI-II & Não \\
\hline $\begin{array}{l}\text { Entrevista + curva } \\
\text { narrativa }\end{array}$ & $\begin{array}{l}\text { Explorar a utilidade do uso do OPHI-II sob a } \\
\text { perspectiva de TOs e seus clientes } \\
\text { diagnosticados com HIV/AIDS }\end{array}$ & Questionário próprio \\
\hline $\begin{array}{l}\text { Entrevista + curva } \\
\text { narrativa }\end{array}$ & $\begin{array}{l}\text { Compreender como o processo terapêutico e a } \\
\text { narrativa do cliente com HIV/AIDS se vinculam } \\
\text { e podem se influenciar mutuamente }\end{array}$ & Diário de campo \\
\hline Entrevista & $\begin{array}{l}\text { Descrever a experiência de vida voltada para o } \\
\text { cuidado de filhos com esquizofrenia }\end{array}$ & Diário de campo \\
\hline Entrevista & $\begin{array}{l}\text { Relacionar os serviços oferecidos e a realidade de } \\
\text { adolescentes em situaçáo de rua }\end{array}$ & Não \\
\hline Entrevista & $\begin{array}{l}\text { Compreender como os fatores pessoais e } \\
\text { ambientais podem influenciar o uso do tempo } \\
\text { de pessoas presas em manicômio judiciário }\end{array}$ & $\begin{array}{l}\text { Australian Time Use } \\
\text { Survey, } \\
\text { Diário de campo }\end{array}$ \\
\hline Entrevista & $\begin{array}{l}\text { Compreender a experiência ocupacional de } \\
\text { pessoas com fadiga crônica }\end{array}$ & Diário de campo \\
\hline Entrevista + escalas & Validar o OPHI-II & Não \\
\hline
\end{tabular}

\section{Discussáo}

Com base nos resultados, as principais finalidades de uso do OPHI-II puderam ser discutidas, levando-se em consideração indicadores bibliométricos das publicaçôes elencadas para a revisão. Assim, os dados são discutidos a partir dos anos de publicação dos estudos, do uso ou não de versóes validadas para a cultura na qual foi aplicado, bem como o local do desenvolvimento da pesquisa. Também é explorada a combinaçâo de outros instrumentos de avaliação com o OPHI-II e a população estudada. Por fim, discutem-se as formas de aplicação do instrumento, que podem ou não incluir as escalas de medida e a curva narrativa.

A elaboração de instrumentos de avaliação, seja para pesquisa ou para a prática, pauta-se na importância do uso de medidas padronizadas, sem as quais a clareza da 
efetividade do processo terapêutico, ou da confiabilidade da pesquisa pode ficar comprometida (Coster \& Mancini, 2015).

Com relação ao ano das publicaçôes, embora a maioria destas tenha ocorrido após 2004, ano em que o instrumento foi publicado em versão impressa, as publicaçóes com a utilização de sua segunda versão começaram em 2001, ano em que este instrumento foi validado por meio do estudo realizado por Kielhofner et al. (2001). Esse dado é importante de ser destacado porque indica que o possível aumento na utilização desse instrumento em pesquisa está relacionado com a sua validação.

A realização de métricas por meio de testes de validaçáo, tanto de novos instrumentos como daqueles que passaram por processo de adaptação transcultural, é essencial para que a qualidade e efetividade do uso da avaliação sejam mantidas em diferentes contextos e populaçôes. Dos 26 artigos selecionados para a revisão, 3 utilizaram a versão adaptada para o hebraico (Ziv \& Roitman, 2008; Bar et al., 2016; Levanon-Erez et al., 2017), e um deles sua versão para o sueco (Sandell et al., 2013), ambas já validadas e testadas para os parâmetros psicométricos em estudos prévios.

Cabe destacar que, embora o OPHI-II já tenha sido validado para o espanhol, nenhum dos estudos encontrados foi conduzido por pesquisadores latino-americanos. Ainda, no caso do Brasil, nenhum estudo foi encontrado. Essa ausência de estudos em nosso país se justifica pelo fato de que são poucos os terapeutas ocupacionais que baseiam a sua prática e pesquisa no MOHO.

Cruz (2018) refere que as razóes para este número reduzido, ou seja, para a pouca adesão tanto ao $\mathrm{MOHO}$ como de outros modelos, estariam relacionadas ao próprio desenvolvimento da terapia ocupacional no Brasil, a qual não foi acompanhada pela criação ou uso consensual de um modelo de prática em específico. No entanto, especificamente em relação à adesão ao $\mathrm{MOHO}$, o autor sinaliza para o processo histórico de formação dos terapeutas ocupacionais no país, na ocasião em que $\mathrm{MOHO}$ foi publicado no Brasil, no início dos anos 90. Cabe destacar que, já na década de 70, em nosso país, os cursos de graduação em terapia ocupacional buscavam modificar a formação que vinha sendo baseada no modelo americano de reabilitação desde seu surgimento em nível técnico, em 1956.

A terapia ocupacional brasileira, do fim dos anos 80 e no transcorrer dos anos 90 , também vivenciou movimentos sociais importantes, como a instauração da democracia, a criação do Sistema Único de Saúde, o Movimento Antimanicomial e as lutas pelos direitos das pessoas com deficiências (Oliver et al., 2018). Como consequência, parte dos terapeutas ocupacionais envolvidos nestes movimentos sociais buscou a constituição de uma terapia ocupacional distanciada dos referenciais internacionais dos quais ela se originou (Cruz, 2018).

Um movimento de polarização envolveu a profissão, sendo, de um lado, terapeutas ocupacionais que investiam nos modelos ou abordagens internacionais focados na ocupação e, de outro, aqueles que se propunham a pensar a terapia ocupacional a partir de referenciais da filosofia, educação, sociologia e antropologia, adotando o termo atividade, que evoluiu para atividade terapêtutica e, posteriormente, atividade humana (Figueiredo et al., 2020; Cruz, 2018; Galheigo et al., 2018).

Desta forma, a carência de instrumentos validados para a cultura brasileira, como é o caso do OPHI-II, constituiria uma consequência resultante deste processo histórico da profissão no Brasil, no qual, segundo Galheigo et al. (2018), desde a criação dos 
primeiros cursos de terapia ocupacional no Brasil, em 1956, os referenciais têm sido incorporados e substituídos de acordo com os contextos e mudanças históricas, sociais e políticas do país. Em conjunto, o ensino, a pesquisa e a extensão, desenvolvidos nas universidades, têm colaborado no processo de inovação dos referenciais e fundamentos para a profissão.

Nesta direção, o processo de adaptação transcultural desse instrumento está em andamento em estudo conduzido no Programa de Pós-Graduação em Terapia Ocupacional da Universidade Federal de São Carlos (UFSCar), o que, futuramente, propiciará o início de investigaçóes que fomentarão o ensino e a formação de terapeutas ocupacionais no país. Tal projeto faz parte de uma iniciativa de um grupo de pesquisa intitulado "Estudos em terapia ocupacional e reabilitação física, tecnologia assistiva e funcionalidade", o qual concentra uma linha de pesquisa com a aplicação de modelos e adaptação transcultural de instrumentos sobre ocupação (Mendes, 2020; Cruz et al., 2019).

Portanto, a pouca adesão histórica do Brasil ao MOHO (Cruz, 2018) fez com que muitos profissionais brasileiros desconhecessem as possibilidades de uso efetivo no processo terapêutico da teoria e de instrumentos baseados no MOHO. Essa realidade é diferente daquela que vem sendo praticada em muitos países, onde o $\mathrm{MOHO}$ tem sido amplamente estudado, divulgado e aplicado, como pôde ser visto nos estudos encontrados, os quais foram realizados em diferentes localidades, como Austrália, Irlanda, Estados Unidos, Israel, Holanda, Suécia e Islândia.

A utilização de instrumentos padronizados para avaliação em terapia ocupacional deve considerar as queixas trazidas pelo cliente e sua condição clínica, sem deixar de relacionar com o desempenho ocupacional do sujeito (Almeida et al., 2015). Vários são os aspectos do domínio da terapia ocupacional que podem sofrer impactos decorrentes de alguma lesão, dentre eles, aqueles apontados pela AOTA como as áreas de ocupação, os padrôes de desempenho, contextos, fatores do cliente, entre outros (Cavalcanti et al., 2015; Carleto et al., 2010). Essa abrangência requer a necessidade de avaliaçóes que contemplem os constructos que são consonantes com os domínios da terapia ocupacional e para a condução do seu processo. Isso repercute em uma variedade de possibilidades de uso de instrumentos de avaliação a serem utilizados no processo terapêutico ocupacional (Figueiredo et al., 2017; Silva \& Martinez, 2002).

Por vezes, o uso de mais de um instrumento de modo concomitante é necessário para ampliar a quantidade de dados coletados, seja para o aprofundamento da pesquisa ou para o planejamento terapêutico, de modo a fornecer informaçôes necessárias para que o cliente possa identificar melhor os seus problemas e pensar nas metas que ele deseja alcançar a partir da intervenção. Esse uso, combinado de um instrumento com outras medidas, pôde ser notado em mais da metade das pesquisas que compóem essa revisão, por exemplo, outros instrumentos padronizados, diários de campo ou questionários elaborados pelos próprios autores.

Considera-se que a variedade de instrumentos de avaliação e de investigação em terapia ocupacional é necessária e de grande contribuição diante da complexidade da ocupação humana enquanto objeto de estudo e intervenção da profissão. Esse uso combinado se justifica, em parte, pela complexidade da ocupação e de seus conceitos relacionados, como o uso de diferentes teorias e os processos volitivos relacionados ao 
fazer, como a exploração, a competência e a realização, por exemplo, conceitos da teoria do comportamento ocupacional que influenciaram o MOHO (Reilly, 1974).

Constatou-se que a populaçáo estudada a partir de dados coletados pelo OPHI-II e analisados com base na teoria do MOHO foi abrangente. Esse achado condiz com o pressuposto inicial da elaboraçáo do instrumento, o qual foi pensado para ser utilizado pelos terapeutas ocupacionais independentemente de sua área de atuação (Hemmingsson et al., 2017). Uma vez que o OPHI-II visa a conhecer melhor o cliente em relação ao seu histórico de desempenho ocupacional, esse instrumento reflete fundamentos conceituais essenciais da terapia ocupacional e da prática centrada no cliente, com seu foco em uma relação terapêutica apoiada no vínculo com ele e baseada na ocupação e não na doença ou deficiência em si (Kielhofner et al., 2004).

Logo, fica evidente que a utilização do OPHI-II não somente é eficaz para favorecer o processo de vinculação entre terapeuta e cliente a partir do conhecimento de sua história, o que também é observado em dois estudos da presente revisão (Apte et al., 2005; Ennals \& Fossey, 2007), mas para o planejamento de uma terapêutica significativa e congruente com o modelo no qual é baseado (Gray \& Fossey, 2003; Farnworth et al., 2004; Ennals \& Fossey, 2007; Ziv \& Roitman, 2008) . O uso de um modelo conceitual da prática, ou, em outras palavras, "teórico-prático", para embasar tanto a intervenção como a pesquisa em terapia ocupacional, fornece meios claros e objetivos, bem como fortalece a filosofia da profissão, uma vez que organiza todo o raciocínio profissional a partir de suas crenças de valores; focados na ocupação (Kielhofner, 2009; Forsyth \& Kielhofner, 2013; Andersen \& Reed, 2017; Cruz, 2020).

Uma vez que o OPHI-II é constituído de entrevista semiestruturada que atua como base para o preenchimento da curva narrativa e das escalas avaliativas, este fornece dados qualitativos e quantitativos. Os meios de utilizaçáo variam e podem ou não incluir o preenchimento das escalas quantitativas e da curva narrativa. Essa flexibilidade possibilita pesquisas com métodos de análise qualitativos, quantitativos ou mistos, o que pôde ser observado pelos resultados desta revisão. Como o preenchimento das escalas necessariamente é precedido pela narrativa da história de vida, e esta pode ser direcionada para diferentes temas de investigação ou focar determinados eventos críticos vivenciados, explica-se o fato de as escolhas metodológicas dos artigos da revisão serem majoritariamente qualitativas (uma característica dos estudos desse modelo), sendo que, dos 26 estudos, apenas 5 eram quantitativos, dois dos quais se referem às validaçóes métricas do instrumento (Kielhofner et al., 2001, 2005).

Essas informaçóes fornecem dados sobre uso do OPHI-II, evidenciando a abrangência populacional, a importância do $\mathrm{MOHO}$ e de suas avaliações no cenário mundial da terapia ocupacional, a possibilidade de aplicação em associação com outras avaliaçóes pertinentes a cada caso de pesquisa ou de terapêutica, e a viabilidade de ser aplicado em diferentes formatos, escolhendo não só o modo de administração do instrumento, mas também sobre a utilização ou não de escalas quantitativas para análise.

Desse modo, percebe-se uma flexibilidade prática do OPHI-II, o qual se mostra potente para conhecer a pessoa, o cliente da terapia, na criação e no fortalecimento do vínculo terapêutico. Além disso, o OPHI-II proporciona um planejamento terapêutico pautado nas experiências individuais significativas de modo objetivo e consistente.

A importância de sua aplicação também se evidencia para a pesquisa, uma vez que tem a capacidade de coletar dados robustos que podem ser estudados à luz da análise 
temática de conteúdo, além dos resultados quantitativos utilizados tanto de modo comparativo quanto para analisar os resultados de uma forma mais objetiva dentro dos serviços que o terapeuta ocupacional pode atuar.

Finalmente, cabe enfatizar que, por meio do uso do OPHI-II, são possíveis de serem analisados muitos constructos e conceitos relacionados aos fundamentos da terapia ocupacional focados na ocupação e centrados no cliente. Segundo Forsyth \& Kielhofner (2013), a teoria do MOHO por si própria incorpora cada cliente individualmente e uma narrativa cultural destes a partir de seus conceitos.

O OPHI-II é um exemplo disso, pois considera culturalmente os valores, interesses e papéis do cliente a partir de uma narrativa etnográfica pela ênfase no método da entrevista. Essa avaliação também é útil para conhecer os pensamentos, sentimentos e ações do cliente que são influenciados culturalmente (Forsyth \& Kielhofner, 2013). Conceitos como forma ocupacional podem ser úteis na identificação sobre como diferentes culturas definem como as ocupaçôes diárias devem ser feitas, logo, conhecer essas formas ocupacionais com base na narrativa do cliente ajuda o terapeuta ocupacional a ser sensível às diferenças culturais que influenciam no modo do cliente para fazer coisas que lhes são significativas (Forsyth \& Kielhofner, 2013).

Compreendendo que a presente revisão pudesse apresentar limitaçôes metodológicas relacionadas aos idiomas considerados e à impossibilidade de incluir todas as bases de dados e periódicos existentes, os autores procuraram ampliar as buscas por meio do uso de diferentes fontes de informação, minimizando o risco de perda de material textual que pudesse ser pertinente às análises do uso do OPHI-II.

Como observado pelos resultados e discussóes apresentadas, o OPHI-II pode ser aplicado com diferentes clientes, e o seu uso pode ser combinado com outros instrumentos de avaliação, pertinentes a cada contexto de prática e de pesquisa. Nota-se ainda que, por ser constituído por 3 partes distintas, a forma de aplicação também é flexível, confirmando a forte característica do modelo no qual o instrumento é baseado, o qual se preocupa com uma prática que tenha processos avaliativos e intervençóes planejadas individualmente para cada pessoa, grupos e coletivos. Assim, fica evidente que o OPHI-II é um potente instrumento para a pesquisa e prática em terapia ocupacional, valorizando a pessoa por meio da prática centrada no cliente, focada na ocupação e baseada em evidência.

\section{Conclusão}

Este trabalho trouxe uma revisão de escopo de estudos que fizeram o uso do instrumento OPHI-II, o qual contribuiu para a construção e ampliação da produção de conhecimento em terapia ocupacional no que se refere à utilização de tal instrumento. Nota-se a existência de uma tendência mundial, em que a profissão tem buscado embasamento teórico-prático no MOHO. No entanto, o Brasil ainda se encontra em um processo anterior, iniciando as pesquisas com o modelo, com a adaptaçáo de seus instrumentos.

Alguns estudos foram excluídos da revisão por não terem utilizado o $\mathrm{OPHI}-\mathrm{II}$ em seu formato original, mas sim como meio de embasar uma nova avaliação. Contudo, essa limitação não influencia nos resultados da revisão, uma vez que esta teve como o 
objetivo descrever características dos estudos que empregaram o instrumento em sua segunda versão - a mais atual no momento.

Pode-se perceber que o uso do instrumento pelas equipes de pesquisa em terapia ocupacional se pauta nos constructos do $\mathrm{MOHO}$, com o foco no cliente e na ocupação. $\mathrm{O}$ uso do instrumento em intervençôes, com o objetivo de uma avaliação inicial para o planejamento terapêutico, também pôde ser observado, uma vez que foram encontrados artigos que relataram a percepção da eficácia do instrumento para o processo terapêutico ocupacional.

Conclui-se que o presente estudo propiciou o conhecimento sobre o uso do OPHIII, por meio da exploração de suas possibilidades e potências tanto para a prática de intervenção como para a pesquisa acadêmica. Espera-se que a disponibilização do OPHI-II para o Brasil possa abrir mais os horizontes para diálogos internacionais sobre o constructo do desempenho ocupacional e a produçáo de conhecimento local que converse sobre o assunto.

\section{Referências}

Almeida, P. H. T. Q., Pontes, T. B., Matheus, J. P. C., Muniz, L. F., \& Mota, L. M. H. (2015). Terapia ocupacional na artrite reumatoide: o que o reumatologista precisa saber? Revista Brasileira de Reumatologia, 55(3), 272-280.

Andersen, L. T., \& Reed, K. L. (2017). The history of occupational therapy: the first century. New York: Slack Incorporated.

Apte, A., Kielhofner, G., Paul-Ward, A., \& Braveman, B. (2005). Therapist's and client's perceptions of the occupational performance history interview. Occupational Therapy in Health Care, 19(1), 173-192. http://dx.doi.org/10.1080/J003v19n01_13.

Arksey, H., \& O'Malley, L. (2005). Scoping studies: towards a methodological framework. International Journal of Social Research Methodology, 8(1), 19-32. http://dx.doi.org/10.1080/1364557032000119616.

Aviles, A., \& Helfrich, C. (2004). Life skill science needs: perspectives of homeless youth. Journal of Youth and Adolescence, 33(4), 331-338.

Bar, M. A., Jarus, T., Wada, M., Rechtman, L., \& Noy, E. (2016). Male-to-female transitions: Implications for occupational performance, health, and life satisfaction. Canadian Journal of Occupational Therapy, 83(2), 72-82. http://dx.doi.org/10.1177/0008417416635346.

Benetton, M. J., \& Lancman, S. (1998). Estudo de confiabilidade e validação da "entrevista da história do desempenho ocupacional”. Revista de Terapia Ocupacional da Universidade de São Paulo, 9(3), 94-104.

Braveman, B., \& Helfrich, C. A. (2001). Occupational identity: exploring the narratives of three men living with AIDS. Journal of Occupational Science, 8(2), 25-31. http://dx.doi.org/10.1080/14427591.2001.9686486.

Braveman, B., Kielhofner, G., Albrecht, G., \& Helfrich, C. (2006). Occupational identity, occupational competence and occupational settings (environment): influences on return to work in men living with HIV/AIDS. Work, 27(3), 267-276.

Cahil, M., Connolly, D., \& Stapleton, T. (2010). Exploring occupational adaptation through the lives of women with multiple sclerosis. British Journal of Occupational Therapy, 73(3), 106-115. http://dx.doi.org/10.4276/030802210X12682330090415.

Callaway, L., Barclay, L., McDonald, R., Farnworth, L., \& Casey, J. (2015). Secondary health conditions experienced by people with spinal cord injury within community living: implications for a National Disability Insurance Scheme. Australian Occupational Therapy Journal, 62(4), 246-254. http://dx.doi.org/10.1111/1440-1630.12206.

Carleto, D. G. S., Souza, A. C., Silva, M., Cruz, D. M. C., \& Andrade, V. S. (2010). Estrutura da prática da Terapia Ocupacional: domínio e processo 2a edição. Revista Triangulo: Ensino, Pesquisa e Extensão, 3(2), 57-147. 
Cavalcanti, A., Dutra, F. C. M. S., \& Elui, V. M. C. (2015). Estrutura da prática da Terapia Ocupacional: domínio \& processo - $3^{a}$ ed. traduzida. Revista de Terapia Ocupacional da Universidade de São Paulo, 26(esp), 1-49. http://dx.doi.org/10.11606/issn.2238-6149.v26iespp1-49

Chaffey, L., \& Fossey, E. (2004). Caring and daily life: occupational experiences of women living with sons diagnosed with schizophrenia. Australian Occupational Therapy Journal, 51(4), 199-207.

Colquhoun, H. L., Levac, D., O’Brien, K. K., Straus, S., Tricco, A. C., Perrier, L., Kastner, M., \& Moher, D. (2014). Scoping reviews: time for clarity in definition, methods, and reporting. Journal of Clinical Epidemiology, 67(12), 1291-1294. http://dx.doi.org/10.1016/j.jclinepi.2014.03.013.

Coster, W. J., \& Mancini, M. C. (2015). Recomendações para a tradução e adaptação. Revista de Terapia Ocupacional da Universidade de São Paulo, 26(1), 50-57. http://dx.doi.org/10.11606/issn.22386149.v26i1p50-57.

Cruz, D. M. C. (2018). Os modelos de Terapia Ocupacional e as possibilidades para a prática e pesquisa no Brasil. Revista Interinstitucional Brasileira de Terapia Ocupacional, 2(3), 504-517.

Cruz, D. M. C. (2020). Prática centrada no cliente: o modelo de ocupação humana e a formulação ocupacional. In L. C. C. Gradim, T. N. Finarde, \& D. C. M. Carrijo. (Eds.), Práticas em Terapia Ocupacional (pp. 41-52). São Paulo: Manole.

Cruz, D. M. C., Parkinson, S., Rodrigues, D. S., Carrijo, D. C. M., Costa, J. D., Martins, E. F., \& Pfeifer, L. I. (2019). Cross-cultural adaptation, face validity and reliability of the Model of Human Occupation Screening Tool to Brazilian Portuguese. Cadernos Brasileiros de Terapia Ocupacional, 27(4), 691-702. http://dx.doi.org/10.4322/2526-8910.ctoao2007.

Davey, H., Imms, C., \& Fossey, E. (2015). “Our child's significant disability shapes our lives”: experiences of family social participation. Disability and Rehabilitation, 37(24), 2264-2271. http://dx.doi.org/10.3109/09638288.2015.1019013.

Donato, H., \& Donato, M. (2019). Etapas na condução de uma revisão sistemática. Acta Medica Portuguesa, 32(3), 227-235. http://dx.doi.org/10.20344/amp.11923.

Ennals, P., \& Fossey, E. (2007). The occupational performance history interview in community mental health case management: consumer and occupational therapist perspectives. Australian Occupational Therapy Journal, 54(1), 11-21. http://dx.doi.org/10.1111/j.1440-1630.2006.00593.x.

Ennals, P., \& Fossey, E. (2009). Using the OPHI-II to support people with mental illness in their recovery. Occupational Therapy in Mental Health, 25(2), 138-150. http://dx.doi.org/10.1080/01642120902859048.

Farnworth, L., Nikitin, L., \& Fossey, E. (2004). Being in a secure forensic psychiatric unit: everyday is the same, killing time or make the most of it. British Journal of Occupational Therapy, 67(10), 430-438.

Figueiredo, L. R. U., Lima, F. F., Mendes, R. S., Marques, N. C. F., Matteuci, M., Almada, H. S., \& Novelli, M. M. P. C. (2017). Adaptação transcultural para a língua portuguesa da Avaliaçáo Cognitiva Dinâmica de Terapia Ocupacional para Crianças (DOTCA-Ch). Cadernos Brasileiros de Terapia Ocupacional, 25(2), 287-296. http://dx.doi.org/10.4322/0104-4931.ctoAO0827.

Figueiredo, M. O., Gomes, L. D., Silva, C. R., \& Martinez, C. M. (2020). A Ocupação e a atividade humana em Terapia Ocupacional: revisão de Escopo na literatura nacional. Cadernos Brasileiros de Terapia Ocupacional, 28(3), 967-982.

Forsyth, K., \& Kielhofner, G. (2013). The Model of Human Occupation: embracing the complexity of occupation by integrating theory into practice and practice into theory. In E. A. S. Duncan (Ed.), Foundations for practice in Occupational Therapy (pp. 51-80). London: Churchill Livingstone.

Galheigo, S. M., Braga, C. P., Arthur, M. A., \& Matsuo, C. M. (2018). Produção de conhecimento, perspectivas e referências teórico-práticas na terapia ocupacional brasileira: marcos e tendências em uma linha do tempo. Cadernos Brasileiros de Terapia Ocupacional, 26(4), 723-738.

http://dx.doi.org/10.4322/2526-8910.ctoAO1773.

Goldstein, K., Kielhofner, G., \& Paul-Ward, A. (2004). Occupational narratives and the therapeutic process. Australian Occupational Therapy Journal, 51(3), 119-124. http://dx.doi.org/10.1111/j.14401630.2004.00443.x. 
Gray, M. L., \& Fossey, E. (2003). Illness experiences and occupations of people with chronic fatigue syndrome. Australian Occupational Therapy Journal, 50(3), 127-136. http://dx.doi.org/10.1046/j.14401630.2003.00336.x.

Hemmingsson, H., Forsyth, K., Haglung, L., Keponen, R., Ekblahd, E., \& Kielhofner, G. (2017). Talking with clients: Assessments that collect informations through interviews. In R. R. Taylor (Ed.), Kielhofner's Model of Human Occupation: theory and application (pp. 188-211). Philadelphia: Wolters Kluwer.

Keller, J., \& Forsyth, K. (2004). The model of human occupational practice. IJOT: Israeli Journal of Occupational Therapy, 13(3), 99-106.

Kielhofner, G. (2009). Conceptual foundations of occupational therapy. Philadelphia: FA Davies Company.

Kielhofner, G., Dobria, L., Forsyth, K., \& Basu, S. (2005). The construction of key-forms for obtaining instantaneous measures from the occupational performance history rating scales. Occupational Therapy Journal of Research: Occupational. Participation and Health, 25(1), 23-31.

Kielhofner, G., Mallinson, T., Crawford, C., Nowak, M., Rigby, M., Henry, A., \& Walwns, D. (2004). Occupational Performance History Interview - Second Version (OPHI-II). Assessment Manual. Chicago: The University of Illinois at Chicago.

Kielhofner, G., Mallinson, T., Forsyth, K., \& Lai, J. S. (2001). Psychometric Properties of the Second Version of the Occupational Performance History Interview (OPHI-II). The American Journal of Occupational Therapy, 55(3), 260-267.

Levanon-Erez, N., Cohen, M., Bar-Ilan, R. T., \& Maeir, A. (2017). Occupational identity of adolescents with ADHD: a mixed methods study. Scandinavian Journal of Occupational Therapy, 24(1), 32-40. http://dx.doi.org/10.1080/11038128.2016.1217927.

Levin, M., \& Helfrich, C. (2004). Mothering role identity and competence among parenting and pregnant homeless adolescents. Journal of Occupational Science, 11(3), 95-104. http://dx.doi.org/10.1080/14427591.2004.9686537.

Levin, M., Kielhofner, G., Braveman, B., \& Fogg, L. (2007). Narrative slope as a predictor of work and other occupational participation. Scandinavian Journal of Occupational Therapy, 14(4), 258-264. http://dx.doi.org/10.1080/11038120701327776.

Mendes, P. V. B. (2020). Adaptação transcultural e propriedades psicométricas do "Occupational Self Assessment" para a língua portuguesa do Brasil (Tese de doutorado). Universidade Federal de São Carlos, São Carlos.

Model of Human Occupation Clearinghouse - MOHO. (2020). Recuperado em 13 de fevereiro de 2020, em: www.moho.uic.edu.

O’Brien, K. K., Colquhoun, H., Levac, D., Baxter, L., Tricco, A. C., Straus, S., Wickerson, L., Nayar, A., Moher, D., \& O'Malley, L. (2016). Advancing scoping study methodology: a web-based survey and consultation of perceptions on terminology, definition and methodological steps. BMC Health Services Research, 16(305), 2-12. http://dx.doi.org/10.1186/s12913-016-1579-z.

O’Connell, M., Farnworth, L., \& Hanson, E. C. (2010). Time use in forensic psychiatry: a naturalistic inquiry into two forensic patients in Australia. International Journal of Forensic Mental Health, 9(2), 101-109. http://dx.doi.org/10.1080/14999013.2010.499558.

O’Donoghue, N., \& McKay, E. (2012). Exploring the impact os sleep apnoea on daily life and occupational engagement. British Journal of Occupational Therapy, 75(11), 509-516. http://dx.doi.org/10.4276/030802212X13522194759932.

Oliver, F. C., Souto, A. C. F., \& Nicolau, S. M. (2018). Terapia Ocupacional em 2019: 50 anos de regulamentação profissional no Brasil. Revisbrato, 2(2), 244-256. http://dx.doi.org/10.47222/25263544.rbto16523.

Pablo, C. G. H. (2015). Modelo de Ocupación Humana. Madrid: Editorial Sintesis

Pablo, C. G. H., Fan, C. W., \& Kielhofner, G. (2017). Dimensions of Doing. In R. R. Taylor (Ed.), Kielhofner's Model of Human Occupation: theory and application (pp. 188-211). Philadelphia: Wolters Kluwer.

Palmadottir, G. (2009). The road to recovery: experience and occupational lives of Icelandic women with breast cancer. Occupational Therapy in Health Care, 23(4), 319-335. http://dx.doi.org/10.3109/07380570903242433. 
Palmadottir, G. (2010). The role of occupational participation and environment among Icelandic women with breast cancer: a qualitative study. Scandinavian Journal of Occupational Therapy, 17(4), 299-307. http://dx.doi.org/10.3109/11038120903302874.

Peters, M. D., Godfrey, C. M., Khalil, H., McInerney, P., Parker, D., \& Soares, C. B. (2015). Guidance for conducting systematic scoping reviews. International Journal of Evidence-Based Healthcare, 13(3), 141146. http://dx.doi.org/10.1097/XEB.0000000000000050.

Reilly, M. (1974). Play as exploratory learning. California: Sage Publications.

Sandell, C., Kjellberg, A., \& Taylor, R. R. (2013). Participating in diagnostic experience: adults with neuropsychiatric disorders. Scandinavian Journal of Occupational Therapy, 20(2), 136-142. http://dx.doi.org/10.3109/11038128.2012.741621.

Silva, D. B. R., \& Martinez, C. M. S. (2002). Modelos de avaliação em terapia ocupacional: estudos dos hábitos funcionais e de auto-suficiência em crianças. Cadernos de Terapia Ocupacional da UFSCar, 10(2), 77-93.

Taylor, R. R. (Ed.). (2017). Kielhofner's Model of Human Occupation: theory and application. Philadelphia: Wolters Kluwer.

Tricco, A. C., Lillie, E., Zarin, W., O’Brien, K., Colquhoun, H., Kastner, M., Levac, D., Ng, C., Sharpe, J. P., Wilson, K., Kenny, M., Warren, R., Wilson, C., Stelfox, H. T., \& Straus, S. E. (2016). A scoping review on the conduct and reporting of scoping reviews. BMC Medical Research Methodology, 16(15), 2 10. http://dx.doi.org/10.1186/s12874-016-0116-4.

Turpin, M., \& Iwama, M. K. (2011). Using occupational therapy models in practice a field guide. Churchill Livingston: Elsevier.

Verhoef, J. A., Roebroeck, M. E., van Schaardenburgh, N., Floothuis, M. C., \& Miedema, H. S. (2014). Improved occupational performance of young adults with a physical disability after a vocational rehabilitation intervention. Journal of Occupational Rehabilitation, 24(1), 42-51. http://dx.doi.org/10.1007/s10926-013-9446-9.

Ziv, N., \& Roitman, D. M. (2008). Addressing the needs of ederly clients whose lives have been compounded by traumatic histories. Occupational Therapy in Health Care, 22(2-3), 85-93. http://dx.doi.org/10.1080/07380570801989507.

\section{Contribuiçáo dos Autores}

Julia Andreza Gorla: concepção e redação do texto, organização e análise dos dados. Cláudia Maria Simôes Martinez: concepção e revisão do texto. Mirela de Oliveira Figueiredo: revisão metodológica e do texto. Daniel Marinho Cezar da Cruz: revisão e redação do texto e orientação quanto à análise dos dados. Todos os autores aprovaram a versão final do texto.

\section{Fonte de Financiamento}

Coordenação de Aperfeiçoamento de Pessoal de Nível Superior (CAPES) - Código 001.

\section{Autor para Correspondência}

Julia Andreza Gorla

e-mail: gorla.ju@gmail.com

\section{Editora de seção}

Profa. Dra. Tatiana Pontes 